\title{
Remarks on the Concept of Dispersion in a Curved Linac
}

\author{
Jean-François Ostiguy \\ Accelerator Physics Center \\ Fermi National Accelerator Laboratory, \\ Batavia, IL $60510^{*}$
}

(Dated: Jan 11 2009)

\begin{abstract}
A next-generation linear collider is expected to span tens of kilometers in length. For various reasons, it may be desirable to house such an accelerator in a tunnel that follows the earth's curvature rather that in a "laser-straight" tunnel. One side effect of opting for a curved linac is the introduction of vertical dispersion. In recent years, much work has been dedicated to understand and evaluate the impact of the presence of dispersion on emittance preservation. While performing simulations with our in-house code (CHEF) we observed a discrepancy between the dispersion function it produces and that computed using other codes in use within the accelerator community. Understanding the origin and the meaning of this discrepancy required a re-examination of the meaning of the concept of dispersion in the context of a linac. The object of this note is to document our findings. We establish that the default dispersion algorithm used by CHEF corresponds to a different, and ultimately more appropriate, definition of the dispersion in presence of acceleration. Not surprisingly, a consistent definition of dispersion restores agreement between codes.
\end{abstract}

*Electronic address: ostiguy@fnal.gov 


\section{DISPERSION IN RINGS AND PASSIVE BEAMLINES}

In a circular accelerator, the dispersion function $\vec{\eta}(s)$ has a clear meaning: it is defined as the first order closed (periodic) orbit deviation arising from a relative change $\delta p / p_{r}$ in the reference momentum $p_{r}$. Thus, one has

$$
\vec{\eta}(s)=\frac{\mathbf{x}\left(s, \frac{\delta p}{p_{r}}\right)-\mathbf{x}(s, 0)}{\frac{\delta p}{p_{r}}}
$$

Recall that in the above, the closed orbit $\mathbf{x}$ is implicitly assumed to be a periodic function of $s$ - the arc length along the reference closed orbit - that is

$$
\mathbf{x}\left(s, \frac{\delta p}{p_{r}}\right)=\mathbf{x}\left(s+L, \frac{\delta p}{p_{r}}\right)
$$

Strictly speaking, true periodicity holds only for an accelerator operating in "storage" (nonaccelerating) mode; however, it remains a good approximation over time scales small with respect to the duration of a complete acceleration cycle. In view of periodicity, the closed orbit function $\mathbf{x}$ is unambiguously specified once a momentum deviation $\frac{\delta p}{p_{r}}$ is specified. As a consequence, at any location $s$ around a ring, one can compute the orbit deviation $\delta \mathbf{x}$ induced by a small change in momentum simply by forming the product of the dispersion and the momentum deviation $\delta p / p_{r}$

$$
\delta \mathbf{x}(s)=\vec{\eta}(s)\left(\delta p / p_{r}\right)
$$

In a passive beamline e.g. a transfer line with no acceleration, there is no a-priori periodicity. Nevertheless, the concept of a dispersion function remains useful. The dispersion can be defined as the difference between an off-momentum trajectory and an on-momentum reference trajectory. Of course, for both trajectories, initial conditions for the transverse coordinates need to be specified. Furthermore, for the off-momentum trajectory, the initial conditions must take into account the initial dispersion, which in the case of a transfer line, is defined unambiguously in the upstream ring.

$$
\mathbf{x}\left(s_{0}, \frac{\delta p}{p_{r}}\right)=\mathbf{x}_{0}+\vec{\eta} \frac{\delta p}{p_{r}}
$$

In practice, beamlines are often designed in such a way that after going through a matching section, the dispersion emerges with values that correspond to initial conditions suitable for periodic oscillations over a sequence of $M$ identical cells. 


\section{LINAC OPTICS}

Just as transfer beamlines, linac lattices are not a-priori optically periodic. Furthermore, in contrast with rings, all acceleration occurs in a single pass and it is essential to consider the properties of the lattice in the presence of acceleration. Typically, the field strength in magnetic elements is scaled in relation to the momentum of a reference particle so that despite acceleration, the situation is optically very similar to that of a passive beamline.

This said, the presence of rf structures always slightly compromises optical periodicity through momentum dependent focusing. Optically, rf accelerating structures introduce focusing in two ways. First, there is the focusing due to the axial mode accelerating electric field "fringes" or longitudinal ends. To first order, in the end regions, the transverse component of the electric field increases linearly from the axis. To the extent that the rf structure fields do not change significantly while the particle traverses it, this is an integral effect that can be described quite well with an angular kick proportional to the accelerating field field and inversely proportional to the momentum. The entrance fringe kick is de-focusing upon entry and focusing upon exit. Note that in view of the momentum increase, the exit focusing is somewhat weaker than the entrance de-focusing, although the asymmetry tends to disappear at high energies. The second type of focusing in rf structures arises from the fact that while the rf structure electric field increases the axial component of the momentum, its transverse component remains unchanged, resulting in a progressive reduction in the angle of the trajectory with respect to the to axis as it traverses the cavity. Both the body and edge focusing in rf structures scale like the inverse of the momentum and therefore, are optically position dependent. In high energy linacs, focusing effects due to rf structures tends to be relatively small and can be considered as a perturbation on the focusing provided by conventional quadrupoles. So in practice, one can devise linac lattices that are optically essentially periodic with corresponding periodic $\beta$ (envelope amplitude) functions.

\section{DISPERSION IN LINACS}

Although dispersion in the context of a linac remains a useful concept, there are subtle differences that are important to appreciate. Obviously, since there is no built-in optical periodicity, one needs, just as for a passive beamline, to specify initial values for the transverse 
coordinates. As we shall see, a more essential distinction between a linac and a storage ring or a passive beamline is that the momentum offset $\delta p / p_{r}$ does not remain constant during acceleration. It is natural to attempt to generalize the definition of dispersion in a linac in the following manner

$$
\eta_{i}(s)=\left[x_{i}\left(x_{0}, x_{0}^{\prime}, y_{0}^{\prime}, y_{0}^{\prime} ; \delta p / p_{r}\right)-x_{i}\left(x_{0}, x_{0}^{\prime}, y_{0}^{\prime}, y_{0}^{\prime} ; 0\right)\right] /\left(\delta p / p_{r}\right)
$$

In this context, $\mathbf{x}\left(\mathbf{x}_{0} ; \delta p / p_{r}\right)$ is a reference trajectory associated with specific initial values of the transverse coordinates and $\delta p / p_{r}$ no longer a constant, but rather is a function of the arc length along the reference trajectory (through its dependence on the machine momentum profile $p_{r}(s)$ ). Note that this definition makes sense only to the extent that synchrotron motion is slow enough that it can be entirely ignored. This is usually the case in a highenergy electron linac, for example.

Our in-house code, CHEF[1], can compute the dispersion in a number of ways. CHEF is not a conventional matrix code; rather, it uses automatic differentiation techniques. By default, dispersion is computed by propagating first order derivatives with respect to the initial state $\delta p / p_{r}$. Without going into details, the procedure is equivalent to the prescription (5), which can also optionally be used explicitly. Curiously, in the presence of acceleration, the dispersion calculated with $\mathrm{CHEF}$ is consistently different from that obtained with other linac codes while in the absence of acceleration (i.e. with the rf structure energy gain set to 0.0) they are in agreement. For some time, we suspected that the discrepancy would be traced to either small differences in the cavity model or to a simple bug. Further testing established that the issue was more fundamental. In particular, numerical tests showed agreement between single particle trajectories computed with CHEF and other codes to be better than seven significant digits. We therefore directed our attention to the specifics of the dispersion calculation as implemented in other codes. Typically, magnetic elements are specified using absolute magnetic field strength. A reference momentum profile is established and subsequently used to scale the magnetic field in all magnetic elements. The dispersion is then computed as follows:

- For each compute a full $6 \times 6$ or a reduced $5 \times 5$ R-matrix (a first order transfer map), using the value of the momentum at the element location to compute the entries.

- propagate the initial dispersion as specified at the entrance of the linac. Explicitly, the state vector that is propagated is $\left(x, x^{\prime}, y, y^{\prime}, s, \delta p / p\right)=\left(\eta_{x 0}, \eta_{x 0}^{\prime}, \eta_{y 0}, \eta_{y 0}^{\prime}, 0,1\right)$ 
It should be noted that once computed, the transfer matrices remain constant. More importantly, the procedure also implicitly assume that the state variable $\delta p / p$ remains constant; hence, it is usually set to 1 . It turns out that this is at the root of the issue. To understand why, it is useful to resort to a conventional differential equation to describe the betatron motion; for simplicity, we consider motion in the $y$-s plane only.

$$
\frac{d^{2} y}{d s^{2}}-\frac{\rho+y}{\rho^{2}}=\frac{B_{x}}{B \rho} \frac{p_{r}}{p}\left(1+\frac{y}{\rho}\right)^{2}
$$

where $\rho$ is the bending radius and $B \rho=p_{r} / e$. Expanding this equation to first order in $y / \rho$ and $\delta=\delta p / p_{r}$ we get

$$
\frac{d^{2} y}{d s^{2}}+\left(\frac{1-\delta}{\rho^{2}(1+\delta)}-\frac{K(s)}{1+\delta}\right) y=\frac{\delta}{\rho(1+\delta)}
$$

where $K(s)=\frac{\partial B_{x}}{\partial y}$. For a particle with $\delta \neq 0$, the solution of the linearized inhomogeneous equation (7) can be expressed as a linear combination of the particular solution (reference trajectory) and a dispersive contribution $\eta(s) \delta$.

$$
y(s)=y_{0}(s)+\eta_{y}(s) \delta
$$

Thus, one has, for the reference trajectory $y_{0}$

$$
\frac{d^{2} y_{0}}{d s^{2}}+K(s) y_{0}=0
$$

and for the dispersive contribution

$$
\frac{d^{2}\left(\eta_{y} \delta(s)\right)}{d s^{2}}+K(s) \eta_{y} \delta(s)=\frac{\delta(s)}{\rho(s)}
$$

Note that in equation (10), the momentum offset $\delta$ cannot be factored out as it would be for a ring at constant energy, because it depends on $s$ through the reference momentum profile $p_{r}(s)$. Note also that the momentum offset profile $\delta(s)$ is determined externally, i.e. the optical equation of motion contains no information about it. Expanding the derivatives, equation (10) takes the form

$$
\delta^{\prime \prime} \eta+2 \delta^{\prime} \eta^{\prime}+\delta \eta^{\prime \prime}+K(s) \eta \delta=\frac{\delta(s)}{\rho(s)}
$$

A case of interest is the case where $\delta p / p_{r}$ is held constant. In practice, this can be realized by launching a particle with some initial value $\delta p / p_{r}$ at $s=0$ after a reduction by the same 
factor of the acceleration gradient of all $\mathrm{rf}$ structures from their nominal (reference) settings. Trivially, one then has $\delta^{\prime \prime}=\delta^{\prime}=0$ and equation (11) becomes

$$
\eta^{\prime \prime}+K(s) \eta=\frac{1}{\rho(s)}
$$

i.e. with $\delta p / p_{r}$ constant, one recovers the standard equation for the dispersion in a ring or a passive beamline. While holding $\delta p / p_{r}$ constant can be useful as a controlled experiment, under operating conditions, $\delta p / p_{r}$ depends on the momentum profile. More realistically, for a linac with a uniform acceleration gradient $g$ one has

$$
\delta(s)=\frac{\delta p_{0}}{p_{r}(s)}=\frac{\delta p_{0}}{p_{r 0}} \frac{1}{\left(1+g s / p_{r 0}\right)}
$$

where $\delta p_{0}$ and $p_{r 0}$ are respectively the momentum offset and the reference momentum at the linac entrance. Clearly, in that case, $\delta^{\prime}$ and $\delta^{\prime \prime}$ no longer vanish. Fig. 1 shows, for a version of the International Collider main linac lattice, the dispersion computed using the default algorithm in CHEF. Fig. 2 shows (left) the dispersion reported by the code Lucretia[2] using its built-in algorithm, and (right) computed, again with Lucretia, with the definition (5) implemented as a custom script.

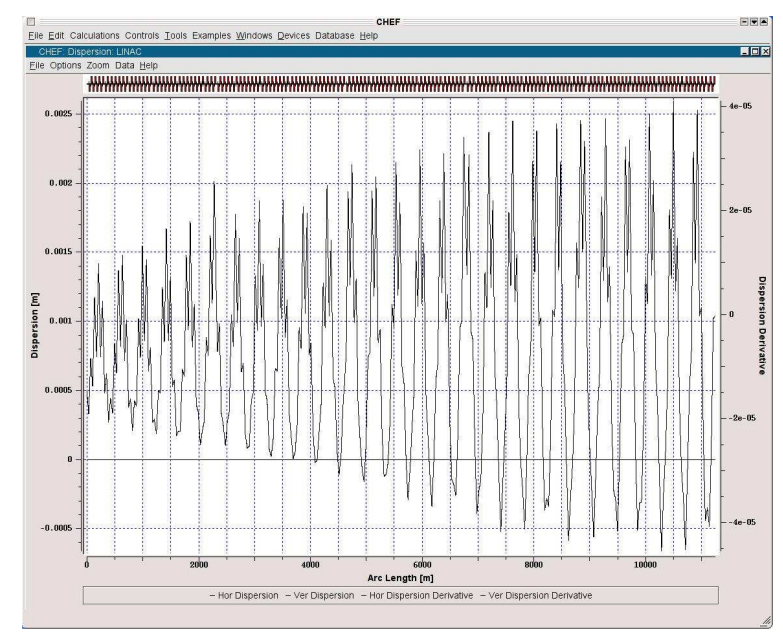

FIG. 1: Dispersion computed by CHEF, for a version of the International Collider main linac lattice.

\section{CONCLUSIONS}

The object of this note was to point out some commonly ignored subtleties in the interpretation of "dispersion" in presence of acceleration. While the dispersion function traditionally 



FIG. 2: Left: Dispersion computed using the default algorithm in Lucretia. Right: Dispersion computed by Lucretia using prescription (5). The vertical units are $10^{-4} \mathrm{~m}$ for the left-hand plot and $10^{-3} \mathrm{~m}$ for the right hand plot. While the horizontal scales are labeled using different units (length and BPM number respectively), they are identical.

computed by matrix codes can be given a meaning, it is important to appreciate that it cannot be used to determine the deviation from the reference orbit at some location $s$ for a given momentum offset $\delta p / p$ at that same location, in the presence of acceleration: for that purpose, the definition (5) must be used. We conclude by stating that the dispersion as computed by CHEF for a high energy linac, is correct. Agreement with other codes is restored when a consistent definition is assumed.

\section{Acknowledgments}

The author is grateful to Dr. Leo Michelotti for helpful discussions and suggestions. Thanks are also due to Dr. Paul Lebrun who initially motivated the author's attention to this issue.

[1] L. Michelotti and J.-F. Ostiguy, CHEF: An Interactive program for Accelerator Optics Calculations., Part. Acc. Conf., Knoxville, TN (2005)

[2] P. Tenenbaum, Lucretia: A Matlab-Based Toolbox for the Modeling and Simulation of SinglePass Electron Beam Transport Systems, Part. Acc. Conf., Knoxville, TN (2005) 